\title{
Comparison of relative versus absolute arm size change as criteria for quantifying breast cancer-related lymphedema: the flaws in current studies and need for universal methodology
}

\author{
Marek Ancukiewicz, \\ Department of Radiation Oncology, Massachusetts General Hospital, 55 Fruit Street, Boston, MA \\ 02114, USA
}

Cynthia L. Miller, Department of Radiation Oncology, Massachusetts General Hospital, 55 Fruit Street, Boston, MA 02114, USA

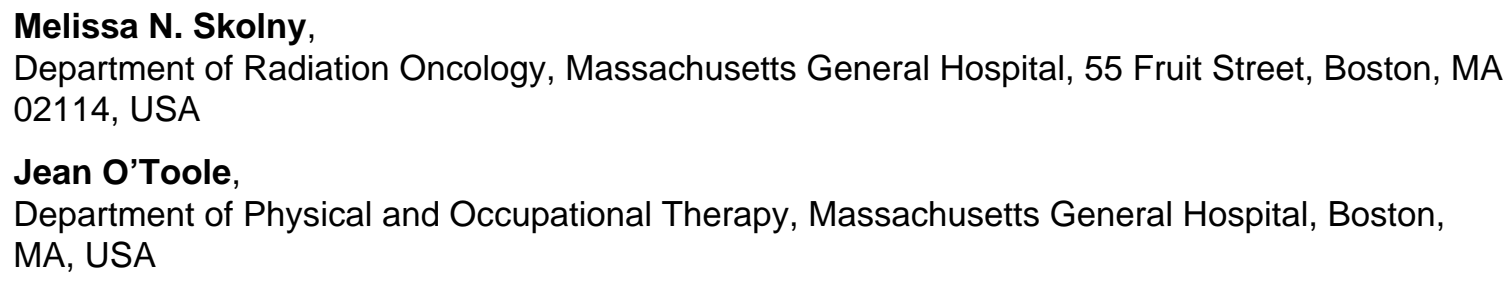

Lauren S. Jammallo, Department of Radiation Oncology, Massachusetts General Hospital, 55 Fruit Street, Boston, MA 02114, USA

Michelle C. Specht, and

Division of Surgical Oncology, Massachusetts General Hospital, Boston, MA, USA

\author{
Alphonse G. Taghian \\ Department of Radiation Oncology, Massachusetts General Hospital, 55 Fruit Street, Boston, MA \\ 02114, USA \\ Alphonse G. Taghian: ataghian@partners.org
}

\begin{abstract}
The purpose of this article is to evaluate arm measurements of breast cancer patients to critically assess absolute change in arm size compared to relative arm volume change as criteria for quantifying breast cancer-related lymphedema (BCRL). We used pre-operative measurements of 677 patients screened for BCRL before and following treatment of unilateral breast cancer at Massachusetts General Hospital between 2005 and 2008 to model the effect of an absolute change in arm size of $200 \mathrm{~mL}$ or $2 \mathrm{~cm}$ compared to relative arm volume change. We also used sequential measurements to analyze temporal variation in unaffected arm volume. Pre-operative arm volumes ranged from 1,270 to $6,873 \mathrm{~mL}$ and correlated strongly (Kendall's $\tau=0.55$ ) with body mass index
\end{abstract}

(c) Springer Science+Business Media, LLC. 2012

Correspondence to: Alphonse G. Taghian, ataghian@partners . org.

Conflicts of interest The authors have no conflicts of interest to disclose. 
(BMI). An absolute arm volume change of $200 \mathrm{~mL}$ corresponded to relative arm volume changes ranging from 2.9 to $15.7 \%$. In a subset of 45 patients, modeling of a $2-\mathrm{cm}$ change in arm circumference predicted relative arm volume changes ranging from 6.0 to $9.8 \%$. Sequential measurements of 124 patients with > 6 measurements demonstrated remarkable temporal variation in unaffected arm volume (median within-patient change $10.5 \%$ ). The magnitude of such fluctuations correlated ( $\tau=0.36, P<0.0001$ ) with pre-operative arm volume, patient weight, and BMI when quantified as absolute volume change, but was independent of these variables when quantified as relative arm volume change $(P>.05)$. Absolute changes in arm size used as criteria for BCRL are correlated with pre-operative and temporal changes in body size. Therefore, utilization of absolute volume or circumference change in clinical trials is flawed because specificity depends strongly on patient body size. Relative arm volume change is independent of body size and should thus be used as the standard criterion for diagnosis of BCRL.

\section{Keywords}

Breast cancer-related lymphedema; Quantification of lymphedema; Arm swelling; Quality of life

\section{Introduction}

Breast cancer-related lymphedema (BCRL) is a sequela of breast cancer treatment (usually surgery or radiotherapy) characterized by swelling of an arm, arms, breast, or trunk, and caused by alteration of normal transport of lymphatic fluid [1]. The lack of a standardized method for quantifying BCRL has been well reported [2-6] and affects the clinical research on diagnosis of BCRL, evaluation of response to treatment, and ability to compare reported data [6-9]. Establishment of a universal standard for quantifying changes in arm size in patients at risk for BCRL will be an important step for progress in diagnosis and treatment of the condition.

As researchers work to generate evidence-based data for early identification and management of BCRL, it is critical that an accurate and widely applicable quantification method be implemented in clinical trials and practice. The consensus document of the international society of lymphology states, "It is desirable the treatment outcomes be reported in a standardized manner to compare and contrast the effectiveness of various treatment protocols" [10]. Presently, the lymphedema framework projects (LFPs) are working on collaborative efforts to set standards for best practice, and the lack of consistency in quantifying and defining BCRL is a common theme in their reports [11, 12]. Armer et al. has demonstrated that in a single patient cohort, the 1-year incidence of BCRL ranges between 21 and $70 \%$ depending on criteria used [13].

The most common objective definitions of BCRL that are the focus of the present report are based on absolute or relative difference in circumference or volume between arms $[4,14$, 15]. Circumferential measurements are commonly performed with tape [16-20], and volumetric measurements are obtained using water displacement [21,22] or optoelectronic perometry [2, 23, 24]. Methods of lymphedema quantification that are based on measurements of arm size are by no means the only ones available: other methods of lymphedema assessment include measurements of arm compressibility (tonometry), MRI, radionuclide scintigraphy, and bioimpedance methods, especially bioimpedance spectroscopy (BIS) [4, 13, 25-27]. Of note, the latter method holds a particular promise because of its practicality, relatively low-upfront cost of equipment, high correlation with perometry [26, 28], and the measurements (i.e., the ratio of extracellular to intra-cellular fluid volumes) which may more directly relate to lymphedema than measurements of the whole arm volume [26, 29]. The comparison of practical utility and diagnostic properties of 
BIS versus state-of-the-art methods based on perometry remains to be performed in larger studies.

The surveys of diagnostic criteria of BCRL in clinical studies indicate that the most popular definitions are based on absolute criteria [20, 30-34] of arm size discrepancy. The diagnosis based on absolute circumference difference (such as $2 \mathrm{~cm}$ ) or volume difference (such as $200 \mathrm{~mL}$ ) has been used in 10 of 11 epidemiological studies reported between 1990 and 2001 and summarized by Cheville et al. [30]; the remaining one study used subjective assessment criteria. The grading criteria of lymphedema based on absolute difference were endorsed by the American Physical Therapy Association and employed in SOMA criterion proposed by Radiation Therapy Oncology Group [30]. In a more recent comprehensive meta-analysis of BCRL studies spanning years from 1944 to 2007 by Tsai et al. [20], the diagnosis based on absolute difference between arms was used in 39 of 47 summarized studies using circumferential measurements and in 9 of 14 summarized studies using volumetric measurements; 4 of 47 and 5 of 14 studies, respectively, used diagnosis based on relative difference between arms (i.e., percent difference); for the remaining 4 studies, it was not clear what criteria were used.

On the other hand, grading lymphedema using relative difference of arm volumes has a long history [35]. The Lymphedema Working Group for Version 3 of Common Toxicity Criteria for Adverse Events of the National Cancer Institute proposed grading of lymphedema using relative difference (i.e., percent difference): $5 \%$ for mild and $10 \%$ for moderate toxicity grade [30]. The rationale for the proposed criteria was that the specific difference such as 2 $\mathrm{cm}$ might be visually undetectable for larger arms but make up a large asymmetry for smaller arms. Despite this, many papers and large clinical trials continue to publish work using absolute criteria to quantify BCRL [3, 5, 14, 15, 36-43]. However, of the studies that do use relative criteria [2, 13, 31, 44-50], few account both for pre-operative asymmetry between arms and changes in size of the contralateral arm over time. The existence of asymmetry between arms has been demonstrated in healthy volunteers, with reports of 3-10 $\%$ volume difference between arms depending on dominance and activity level [51]; similarly, Ward et al. found a significant difference in the raw impedance values between arms, reflecting an asymmetry which the authors attributed to the effects of arm dominance [52]. The importance of pre-operative measurements, as well as compensation for changes in the size of both arms [reflecting temporal body mass index (BMI) fluctuations] has been suggested [2, 14, 23, 47, 49].

In a previous report, we described a protocol ensuring accurate measurements of arm volumes using perometry and proposed-based on the statistical analysis of several variation sources in such measurements - the Relative Volume Change (RVC) formula that accounted for both pre-operative measurement and for changes in the affected and unaffected arms over time [24]: $\mathrm{RVC}=\left(A_{2} U_{1} / U_{2} A_{1}\right)-1$, where $A_{1}$ and $A_{2}$ are arm volumes of the treated arm at pre-operative baseline and follow-up and $U_{1}$ and $U_{2}$ are arm volumes of the untreated arm at the corresponding time points.

The aim of this article was to evaluate the effect of an absolute change in arm size of 200 $\mathrm{mL}$ or $2 \mathrm{~cm}$ compared with relative arm volume change as criteria for defining BCRL. We sought to determine whether body size was a significant factor contributing to absolute and relative arm size changes. 


\section{Methods}

\section{Patients}

We used bilateral perometer arm measurements of 677 consecutive patients screened for lymphedema pre-operatively and following surgical treatment of unilateral breast cancer at Massachusetts General Hospital between 2005 and 2008; the study was approved by the Institutional Review Board. The details of measurement protocol, data collection, as well as patient characteristics have been previously described [24].

\section{Analysis}

Using pre-operative arm measurements of patients in this cohort, we calculated the effect of $200 \mathrm{~mL}$ arm volume change compared with relative arm volume change, assuming that the volume of the opposite arm remains unchanged. In addition, we modeled the effect of a change in arm circumference by $2 \mathrm{~cm}$ in a sample of 45 patients compared with relative arm volume change. This was done using pre-operative arm measurements assuming the following: the 2-cm change occurred in the middle of arm, there was no change at both arm ends, and in-between point changes were obtained using linear interpolation. Finally, we analyzed the within-patient variation of arm volumes over the course of follow-up, in a subset of 124 patients with 6-13 sequential measurements. The analysis was performed in the unaffected arm only (opposite to the side of breast surgery) in order to minimize the potential effect of lymphedema on measurement variation.

\section{Statistical methods}

Histograms were used for graphical assessment of statistical distribution of preoperative arm volumes. The within-patient temporal variation of absolute sequential arm volume change was evaluated in a subset of 124 patients with 6-13 sequential measurements using two measures of variation: standard deviation and the range, the difference between the largest and the smallest measurement. Kendall's correlation coefficients were calculated, and Kendall's test [53] was used as a non-parametric test of correlation.

\section{Results}

Preoperative unaffected arm volumes exhibited an approximately lognormal distribution (Fig. 1) with a median of 2,560 mL and a range from 1,270 to $6,873 \mathrm{~mL}$ (5.4-fold variation). There was a strong correlation of unaffected arm volume with patient body weight (Kendall's $\tau=0.65, P<0.0001$ ) and BMI (Kendall's $\tau=0.55, P<0.0001$ ).

For all 677 patients, an absolute change in arm volume by $200 \mathrm{~mL}$ corresponded to a relative arm volume change of $15.7 \%$ for pre-operative arm volume of $1,270 \mathrm{~mL}$ and $2.9 \%$ for preoperative arm volume of $6,873 \mathrm{~mL}$. The median relative arm volume change resulting from a 200-mL change in arm volume was $7.7 \%$ (Fig. 2).

In a subset of 45 patients, a local 2 -cm change in arm circumference corresponded to a relative arm volume change of $9.8 \%$ for pre-operative arm volume of $1,799 \mathrm{~mL}$ and $6.0 \%$ for preoperative arm volume of $4,376 \mathrm{~mL}$. The median relative arm volume change resulting from a circumference change of $2 \mathrm{~cm}$ was $7.5 \%$ (Fig. 3).

In a subset of 124 patients with 6 or more sequential measurements (range of 6-13), the range of longitudinal (within-patient) variation of absolute volume change of the unaffected arm is correlated with pre-operative arm volume (Kendall's $\tau=0.34, P<0.0001$ ), patient weight (Kendall's $\tau=0.41, P<0.0001$ ), and patient BMI (Kendall's $\tau=0.36, P<0.0001$ ). In contrast, the range of variation in relative arm volume changes, though substantial in 
magnitude (median range in this subpopulation was $10.5 \%$ ), was not correlated with preoperative arm volume, patient weight, or patient BMI $(P>0.05$ for all). Similar results were obtained for standard deviation of longitudinal variation: for absolute volume changes, the standard deviation is correlated with preoperative arm volume, patient weight, and patient BMI $(P<0.0001$ for all); however, the correlations were not statistically significant for relative arm volume changes $(P>0.05$ for all).

Pre-operative asymmetry between arms was often observed. For 30.0 and $8.1 \%$ of patients in our cohort, the absolute volume difference between arms at the preoperative measurement exceeded 100 and $200 \mathrm{~mL}$, respectively (Fig. 4).

\section{Discussion}

Our analysis demonstrates that an absolute change in arm size of $200 \mathrm{~mL}$ or $2 \mathrm{~cm}$ corresponds to a considerable range of relative arm volume changes depending on initial body size. We also found that temporal change in the absolute volume of the unaffected arm is correlated with body size, but relative arm volume change is not. Therefore, relative arm volume change can be used to accurately quantify BCRL in a range of body sizes and with changes in body size over time.

We found that the arm size of patients diagnosed with breast cancer varies within a broad range: the ratio of largest to smallest arm volume in our patient sample was more than 5:1. With such variation, the visual—and likely the functional—impact of the same absolute increase in arm size $(200 \mathrm{~mL}$ or $2 \mathrm{~cm})$ is very different in patients with small and large arms. For example, an increase of $200 \mathrm{~mL}$ in arm volume corresponds to 15.4, and $3.3 \%$ increase in relative arm volume for arms of 1,300 and $6,000 \mathrm{~mL}$ volumes, respectively. Thus, the absolute and relative criteria for diagnosis of BCRL are mutually inconsistent in populations with heterogeneous arm sizes.

We found compelling evidence that absolute arm volume changes over time of the unaffected arm correlate with body size measures (including initial patient BMI, weight, and pre-operative arm volume). However, we found no evidence that temporal relative arm volume changes correlate with these body size measures. In other words, the fluctuations in arm volume have larger magnitude for larger patients. Therefore, we can expect that when the diagnosis of BCRL is based on absolute criteria, the specificity depends on body size. That is, for larger patients, the chances of a false positive diagnosis of BCRL—crossing at random a threshold of a specific size, for example, $2 \mathrm{~cm}$ or $200 \mathrm{~mL}$ - are higher. In contrast, when using relative arm volume change, the magnitude of random variation does not depend on patient body size.

One consequence of this association is that the prevalence of BCRL diagnosed using absolute criteria necessarily correlates with patient BMI. This association has, indeed, been reported by studies using absolute criteria [15, 44, 54]. However, more frequent diagnosis of BCRL for patients with larger body size reflects, at least partially, a higher likelihood of false positive findings in obese patients, as arm size fluctuations unrelated to lymphedema have higher magnitude in such patients. Obesity may very well be a real risk factor for the development of BCRL; however, a study of such correlation must use a diagnosis based on relative arm volume change criterion, otherwise it is not clear if the findings reflect merely the statistical artifact of increased false positive rate for patients with higher BMI.

There may be other significant consequences of using body size-dependent diagnosis of BCRL. For example, smaller patients might be under-diagnosed and therefore not receive proper treatment. For such patients, the increase of $2 \mathrm{~cm}$ in circumference or $200 \mathrm{~mL}$ in volume may represent up to $15 \%$ increase in relative arm volume; these patients may miss 
the opportunity for potentially effective treatment had they been diagnosed at low level of relative arm volume change such as 5-10\%. Given that BCRL is a potentially devastating condition that may require long-term management and is associated with risk of functional, psychosocial, and economic detriments, under-diagnosis may greatly compromise a breast cancer patient's quality of life in the years after treatment [55-61]. In contrast, patients with large initial arm size may be falsely diagnosed with lymphedema based upon absolute changes which correspond to only a $3 \%$ relative arm volume increase. These patients would be unnecessarily over-treated, imposing undue burden on the patient while misappropriating limited healthcare resources. Furthermore, the mislabeling of patients as having lymphedema when they do not could result in significant detriment to their quality of life.

The results of epidemiological or clinical studies of BCRL using diagnosis based on absolute criterion such as a difference or change of $2 \mathrm{~cm}$ or $200 \mathrm{~mL}$ may not be accurately generalized to populations with different distributions of patient body size. As illustrated in the meta-analysis by Tsai et al. [20, 37, 62], criteria based on absolute difference in arm size from circumferential or volumetric measurements are frequent in the literature. We find it troublesome when a flawed absolute criterion is used in important studies that are likely to impact and possibly change the standard of care regarding treatment of lymphedema. One example is the ACOSOG Z0011 trial, comparing incidence of BCRL after axillary lymph node dissection (ALND) or sentinel lymph node biopsy (SLNB) alone, which has had a significant impact on clinical practice. In this study, BCRL was defined as a $2 \mathrm{~cm}$ or greater postoperative increase in ipsilateral arm measured at $10 \mathrm{~cm}$ proximal to the medial epicondyle, compared with contralateral arm measurements. The data showed that the risk of BCRL at 12 months was $11 \%$ or $6 \%$ for patients with ALND or SLNB alone, respectively $(P=0.07)$. Another example of the use of absolute criteria is in the work of McLaughlin et al., who reported BCRL incidence in 936 breast cancer patients comparing those with ALND and SLNB. They utilized a 2-cm absolute change postoperatively measured $10 \mathrm{~cm}$ above the olecranon and $5 \mathrm{~cm}$ below to indicate the presence of BCRL. The results demonstrated that $6 \%$ of SLNB patients had lymphedema compared to $16 \%$ of those with ALND. If these rates are used to counsel patients when the type of axillary surgery is discussed, it is possible that the risk of mild lymphedema for patients with small arms might be underestimated and vice versa for patients with large arms. Therefore, more accurate rates of BCRL occurring after different treatment modalities, particularly type of axillary surgery, are needed. Table 1 summarizes 10 recent randomized studies where lymphedema data were analyzed, of which 6 used diagnostic criteria based on absolute change in either volume or circumference. Whenever feasible, we suggest re-analysis of these results using relative arm volume change as the criterion.

Data from this analysis should provide evidence that lymphedema should be quantified as a relative arm volume change rather than absolute changes in arm size. Given that $30 \%$ of patients in our series had an asymmetry in arm volume preoperatively exceeding $100 \mathrm{~mL}$, one should adjust for pre-operative arm measurements when quantifying lymphedema. Changes in size of the contralateral arm should also be used as a control to account for temporal fluctuations in BMI. We therefore suggest use of the Relative Volume Change $(\mathrm{RVC})$ formula as a criterion for lymphedema $\left(\mathrm{RVC}=\left(A_{2} U_{1}\right) /\left(U_{2} A_{1}\right)-1\right.$, where $A_{1}, A_{2}$ are arm volumes on the side of treated breast at two different time points and $U_{1}, U_{2}$ are arm volumes on the opposite side) [24]. Widespread implementation of this formula for quantification of arm volume changes related to BCRL will facilitate comparison of data collected from clinical trials and result in a better understanding of lymphedema incidence and treatment outcome. 


\section{Acknowledgments}

The project described was supported by Award no. R01CA139118 (AGT) and Award no. P50CA089393 (AGT) from the National Cancer Institute. The content is solely the responsibility of the authors and does not necessarily represent the official views of the National Cancer Institute or the National Institutes of Health.

\section{References}

1. Murdaca G, Cagnati P, Gulli R, Spano F, Puppo F, Campisi C, Boccardo F. Current views on diagnostic approach and treatment of lymphedema. Am J Med. 2012; 125(2):134-140.10.1016/ j.amjmed.2011.06.032 [PubMed: 22269614]

2. Cormier JN, Xing Y, Zaniletti I, Askew RL, Stewart BR, Armer JM. Minimal limb volume change has a significant impact on breast cancer survivors. Lymphology. 2009; 42(4):161-175. [PubMed: 20218084]

3. Ahmed RL, Thomas W, Yee D, Schmitz KH. Randomized controlled trial of weight training and lymphedema in breast cancer survivors. J Clin Oncol: Off J Am Soc Clin Oncol. 2006; 24(18): 2765-2772.

4. Armer JM. The problem of post-breast cancer lymphedema: impact and measurement issues. Cancer Invest. 2005; 23(1):76-83. [PubMed: 15779870]

5. Smoot BJ, Wong JF, Dodd MJ. Comparison of diagnostic accuracy of clinical measures of breast cancer-related lymphedema: area under the curve. Arch Phys Med Rehabil. 2011; 92(4):603-610. [PubMed: 21440706]

6. Ward L. Is BIS ready for prime time as the gold standard measure? J Lymphoedema. 2009; 4(2):5256.

7. Paskett ED. Breast cancer-related lymphedema: attention to a significant problem resulting from cancer diagnosis. J Clin Oncol. 2008; 26(35):5689-5696. [PubMed: 19001331]

8. Clark B, Sitzia J, Harlow W. Incidence and risk of arm oedema following treatment for breast cancer: a three-year follow-up study. QJM: Month J Assoc Phys. 2005; 98(5):343-348.

9. Hayes S, Janda M, Cornish B, Battistutta D, Newman B. Lymphedema secondary to breast cancer: how choice of measure influences diagnosis, prevalence, and identifiable risk factors. Lymphology. 2008; 41(1):18-28. [PubMed: 18581955]

10. International Society of Lymphology. The diagnosis and treatment of peripheral lymphedema. 2009 Consensus document of the international society of lymphology. Lymphology. 2009; 42:5160. [PubMed: 19725269]

11. Armer JM, Stewart BR, Shook RP. 30-Month post-breast cancer treatment lymphoedema. J Lymphoedema. 2009; 4(1):14-18. [PubMed: 20182653]

12. Cormier JN, Askew RL, Mungovan KS, Xing Y, Ross MI, Armer JM. Lymphedema beyond breast cancer: a systematic review and meta-analysis of cancer-related secondary lymphedema. Cancer. 2010; 116(22):5138-5149.10.1002/cncr.25458 [PubMed: 20665892]

13. Armer JM, Stewart BR. A comparison of four diagnostic criteria for lymphedema in a post-breast cancer population. Lymphat Res Biol. 2005; 3(4):208-217. [PubMed: 16379589]

14. Boccardo FM, Ansaldi F, Bellini C, Accogli S, Taddei G, Murdaca G, Campisi CC, Villa G, Icardi G, Durando P, Puppo F, Campisi C. Prospective evaluation of a prevention protocol for lymphedema following surgery for breast cancer. Lymphology. 2009; 42(1):1-9. [PubMed: 19499762]

15. Bar Ad V, Cheville A, Solin LJ, Dutta P, Both S, Harris EE. Time course of mild arm lymphedema after breast conservation treatment for early-stage breast cancer. Int J Radiat Oncol Biol Phys. 2010; 76(1):85-90. [PubMed: 19427748]

16. Schunemann H, Willich N. Lymphedema after breast carcinoma. A study of 5868 cases. Dtsch Med Wochenschr. 1997; 122(17):536-541. [PubMed: 9190300]

17. Coen JJ, Taghian AG, Kachnic LA, Assaad SI, Powell SN. Risk of lymphedema after regional nodal irradiation with breast conservation therapy. Int J Radiat Oncol Biol Phys. 2003; 55(5): 1209-1215. [PubMed: 12654429] 
18. Herd-Smith A, Russo A, Muraca MG, Del Turco MR, Cardona G. Prognostic factors for lymphedema after primary treatment of breast carcinoma. Cancer. 2001; 92(7):1783-1787. [PubMed: 11745250]

19. Wilke LG, McCall LM, Posther KE, Whitworth PW, Reintgen DS, Leitch AM, Gabram SG, Lucci A, Cox CE, Hunt KK, Herndon JE, Giuliano AE. Surgical complications associated with sentinel lymph node biopsy: results from a prospective international cooperative group trial. Ann Surg Oncol. 2006; 13(4):491-500. [PubMed: 16514477]

20. Tsai RJ, Dennis LK, Lynch CF, Snetselaar LG, Zamba GK, Scott-Conner C. The risk of developing arm lymphedema among breast cancer survivors: a meta-analysis of treatment factors. Ann Surg Oncol. 2009; 16(7):1959-1972. [PubMed: 19365624]

21. Edwards TL. Prevalence and aetiology of lymphoedema after breast cancer treatment in southern Tasmania. Aust NZ J Surg. 2000; 70(6):412-418.

22. Beaulac SM, McNair LA, Scott TE, LaMorte WW, Kavanah MT. Lymphedema and quality of life in survivors of early-stage breast cancer. Arch Surg. 2002; 137(11):1253-1257. [PubMed: 12413312]

23. Stout Gergich NL, Pfalzer LA, McGarvey C, Springer B, Gerber LH, Soballe P. Preoperative assessment enables the early diagnosis and successful treatment of lymphedema. Cancer. 2008; 112(12):2809-2819. [PubMed: 18428212]

24. Ancukiewicz M, Russell TA, Otoole J, Specht M, Singer M, Kelada A, Murphy CD, Pogachar J, Gioioso V, Patel M, Skolny M, Smith BL, Taghian AG. Standardized method for quantification of developing lymphedema in patients treated for breast cancer. Int J Radiat Oncol Biol Phys. 2011; 79(5):1436-1443. [PubMed: 20605339]

25. Gerber LH. A review of measures of lymphedema. Cancer. 1998; 83(12 Suppl American):28032804. [PubMed: 9874401]

26. Ward LC, Czerniec S, Kilbreath SL. Operational equivalence of bioimpedance indices and perometry for the assessment of unilateral arm lymphedema. Lymphat Res Biol. 2009; 7(2):81-85. [PubMed: 19522677]

27. Rockson SG. Bioimpedance analysis in the assessment of lymphoedema diagnosis and management. J Lymphoedema. 2007; 2(1):44-48.

28. Jain MS, Danoff JV, Paul SM. Correlation between bio-electrical spectroscopy and perometry in assessment of upper extremity swelling. Lymphology. 2010; 43(2):85-94. [PubMed: 20848996]

29. Czerniec SA, Ward LC, Refshauge KM, Beith J, Lee MJ, York S, Kilbreath SL. Assessment of breast cancer-related arm lymphedema-comparison of physical measurement methods and selfreport. Cancer Invest. 2010; 28(1):54-62. [PubMed: 19916749]

30. Cheville AL, McGarvey CL, Petrek JA, Russo SA, Thiadens SR, Taylor ME. The grading of lymphedema in oncology clinical trials. Semin Radiat Oncol. 2003; 13(3):214-225. [PubMed: 12903011]

31. Schmitz KH, Ahmed RL, Troxel AB, Cheville A, Lewis-Grant L, Smith R, Bryan CJ, WilliamsSmith CT, Chittams J. Weight lifting for women at risk for breast cancer-related lymphedema: a randomized trial. JAMA. 2010; 304(24):2699-2705. [PubMed: 21148134]

32. Vignes S, Porcher R, Arrault M, Dupuy A. Long-term management of breast cancer-related lymphedema after intensive decongestive physiotherapy. Breast Cancer Res Treat. 2007; 101(3): 285-290. [PubMed: 16826318]

33. Devoogdt N, Van Kampen M, Geraerts I, Coremans T, Christiaens MR. Different physical treatment modalities for lymphoedema developing after axillary lymph node dissection for breast cancer: a review. Eur J Obstet Gynecol Reprod Biol. 2009; 149(1):3-9. [PubMed: 20018422]

34. Kaya T, Karatepe AG, Gunaydn R, Yetis H, Uslu A. Disability and health-related quality of life after breast cancer surgery: relation to impairments. South Med J. 2010; 103(1):37-41.10.1097/ SMJ.0b013e3181c38c41 [PubMed: 19996840]

35. Saaristo AM, Niemi TS, Viitanen TP, Tervala TV, Hartiala P, Suominen EA. Microvascular breast reconstruction and lymph node transfer for postmastectomy lymphedema patients. Ann Surg. 2012; 255(3):468-473.10.1097/SLA.0b013e3182426757 [PubMed: 22233832]

36. McLaughlin SA, Wright MJ, Morris KT, Giron GL, Sampson MR, Brockway JP, Hurley KE, Riedel ER, Van Zee KJ. Prevalence of lymphedema in women with breast cancer 5 years after 
sentinel lymph node biopsy or axillary dissection: objective measurements. J Clin Oncol: Off J Am Soc Clin Oncol. 2008; 26(32):5213-5219.

37. Lucci A, McCall LM, Beitsch PD, Whitworth PW, Reintgen DS, Blumencranz PW, Leitch AM, Saha S, Hunt KK, Giuliano AE. Surgical complications associated with sentinel lymph node dissection (SLND) plus axillary lymph node dissection compared with SLND alone in the American College of Surgeons Oncology Group Trial Z0011. J Clin Oncol. 2007; 25(24):36573663. [PubMed: 17485711]

38. Devoogdt N, Christiaens MR, Geraerts I, Truijen S, Smeets A, Leunen K, Neven P, Van Kampen M. Effect of manual lymph drainage in addition to guidelines and exercise therapy on arm lymphoedema related to breast cancer: randomised controlled trial. BMJ. 2011; 343:d5326d5326.10.1136/bmj.d5326 [PubMed: 21885537]

39. Kim do S, Sim YJ, Jeong HJ, Kim GC. Effect of active resistive exercise on breast cancer-related lymphedema: a randomized controlled trial. Arch Phys Med Rehabil. 2010; 91(12):1844-1848. [PubMed: 21112424]

40. Omar MTA, Ebid A, El Morsy AM. Treatment of post-mastectomy lymphedema with laser therapy: double blind placebo control randomized study. J Surg Res. 2011; 165(1):82-90. [PubMed: 20538293]

41. Kozanoglu E, Basaran S, Paydas S, Sarpel T. Efficacy of pneumatic compression and low-level laser therapy in the treatment of postmastectomy lymphoedema: a randomized controlled trial. Clin Rehabil. 2009; 23(2):117-124. [PubMed: 19164399]

42. van der Veen P, De Voogdt N, Lievens P, Duquet W, Lamote J, Sacre R. Lymphedema development following breast cancer surgery with full axillary resection. Lymphology. 2004; 37(4):206-208. [PubMed: 15693539]

43. Goldberg JI, Wiechmann LI, Riedel ER, Morrow M, Van Zee KJ. Morbidity of sentinel node biopsy in breast cancer: the relationship between the number of excised lymph nodes and lymphedema. Ann Surg Oncol. 2010; 17(12):3278-3286. [PubMed: 20574774]

44. Soran A, Wu WC, Dirican A, Johnson R, Andacoglu O, Wilson J. Estimating the probability of lymphedema after breast cancer surgery. Am J Clin Oncol. 2010; 34(5):506-510. [PubMed: 21127413]

45. Ashikaga T, Krag DN, Land SR, Julian TB, Anderson SJ, Brown AM, Skelly JM, Harlow SP, Weaver DL, Mamounas EP, Costantino JP, Wolmark N. Morbidity results from the NSABP B-32 trial comparing sentinel lymph node dissection versus axillary dissection. J Surg Oncol. 2010; 102(2):111-118. [PubMed: 20648579]

46. Kosir MA, Rymal C, Koppolu P, Hryniuk L, Darga L, Du W, Rice V, Mood D, Shakoor S, Wang W, Bedoyan J, Aref A, Biernat L, Northouse L. Surgical outcomes after breast cancer surgery: measuring acute lymphedema. J Surg Res. 2001; 95(2):147-151. [PubMed: 11162038]

47. Armer JM, Stewart BR. Post-breast cancer lymphedema: incidence increases from 12 to 30 to 60 months. Lymphology. 2010; 43(3):118-127. [PubMed: 21226414]

48. Smith MJ, Gill PG, Wetzig N, Sourjina T, Gebski V, Ung O, Campbell I, Kollias J, Coskinas X, Macphee A, Young L, Simes RJ, Stockler MR. Comparing patients' and clinicians' assessment of outcomes in a randomised trial of sentinel node biopsy for breast cancer (the RACS SNAC trial). Breast Cancer Res Treat. 2009; 117(1):99-109. [PubMed: 18925434]

49. Mahamaneerat WK, Shyu CR, Stewart BR, Armer JM. Breast cancer treatment, BMI, post-op swelling/lymphoedema. J Lymphoedema. 2008; 3(2):38-44. [PubMed: 20657749]

50. Dayes IS, Levine MN, Julian JA, Pritchard KI, D'Souza DP, Kligman L, Reise D, Wiernikowski JA, Bonilla L, Whelan TJ. Lymphedema in women with breast cancer: characteristics of patients screened for a randomized trial. Breast Cancer Res Treat. 2008; 110(2):337-342. [PubMed: 17851756]

51. Gebruers N, Truijen S, Engelborghs S, De Deyn PP. Volumetric evaluation of upper extremities in 250 healthy persons. Clin Physiol Funct Imaging. 2007; 27(1):17-22. [PubMed: 17204033]

52. Chachaj A, Malyszczak K, Pyszel K, Lukas J, Tarkowski R, Pudelko M, Andrzejak R, Szuba A. Physical and psychological impairments of women with upper limb lymphedema following breast cancer treatment. Psycho-Oncology. 2012; 19(3):299-305.10.1002/pon.1573 [PubMed: 19399782]

53. Hollander, M.; Wolfe, DA. Nonparametric statistical methods. Wiley; New York: 1999. 
54. Helyer LK, Varnic M, Le LW, Leong W, McCready D. Obesity is a risk factor for developing postoperative lymphedema in breast cancer patients. Breast J. 2010; 16(1):48-54. [PubMed: 19889169]

55. Bernas, MjARL. Lymphedema: how do we diagnose and reduce the risk of this dreaded complication of breast cancer treatment? Curr Breast Cancer Rep. 2010; 2:53-58.

56. Lee TS, Kilbreath SL, Sullivan G, et al. Factors that affect intention to avoid strenuous arm activity after breast cancer surgery. Oncol Nurs Forum. 2009; 36(4):454-462. [PubMed: 19581236]

57. Kwan ML, Darbinian J, Schmitz KH, Citron R, Partee P, Kutner SE, Kushi LH. Risk factors for lymphedema in a prospective breast cancer survivorship study: the pathways study. Arch Surg. 2010; 145(11):1055-1063. [PubMed: 21079093]

58. Yang EJ, Park WB, Seo KS, et al. Longitudinal change of treatment-related upper limb dysfunction and its impact on late dysfunction in breast cancer survivors: a prospective cohort study. J Surg Oncol. 2010; 101(1):84-91. [PubMed: 19924721]

59. Amer MS, Li J, O’Regan DJ, Steele DS, Porter KE, Sivaprasadarao A, Beech DJ. Translocon closure to $\mathrm{Ca}^{2+}$ leak in proliferating vascular smooth muscle cells. Am J Physiol Heart Circ Physiol. 2009; 296(4):H910-H916.10.1152/ajpheart.00984. 2008 [PubMed: 19218505]

60. Chachaj A, Malyszczak K, Pyszel K, Lukas J, Tarkowski R, Pudelko M, Andrzejak R, Szuba A. Physical and psychological impairments of women with upper limb lymphedema following breast cancer treatment. Psychooncology. 2010; 19(3):299-305. [PubMed: 19399782]

61. Stout NL, Pfalzer LA, Levy E, McGarvey C, Springer B, Gerber LH, Soballe P. Segmental limb volume change as a predictor of the onset of lymphedema in women with early breast cancer. PM \& R: J Inj, Funct, Rehabil. 2011; 3(12):1098-1105.

62. Caudle AS, Hunt KK, Kuerer HM, Meric-Bernstam F, Lucci A, Bedrosian I, Babiera GV, Hwang RF, Ross MI, Feig BW, Hoffman K, Litton JK, Sahin AA, Yang W, Hortobagyi GN, Buchholz TA, Mittendorf EA. Multidisciplinary considerations in the implementation of the findings from the American College of Surgeons Oncology Group (ACOSOG) Z0011 study: a practice-changing trial. Ann Surg Oncol. 2011; 18(9):2407-2412. [PubMed: 21327455] 


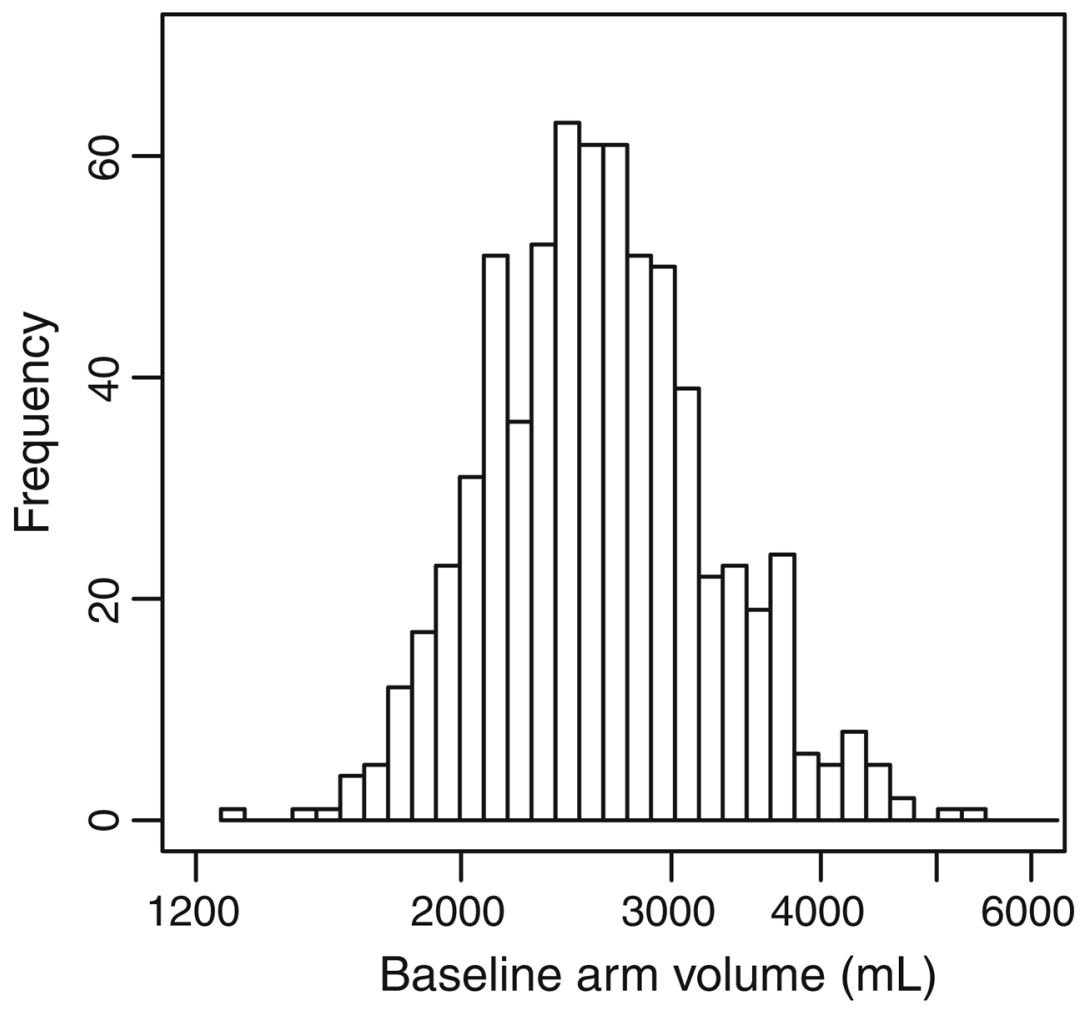

Fig. 1.

Histogram of pre-operative unaffected arm volumes in 677 patients 


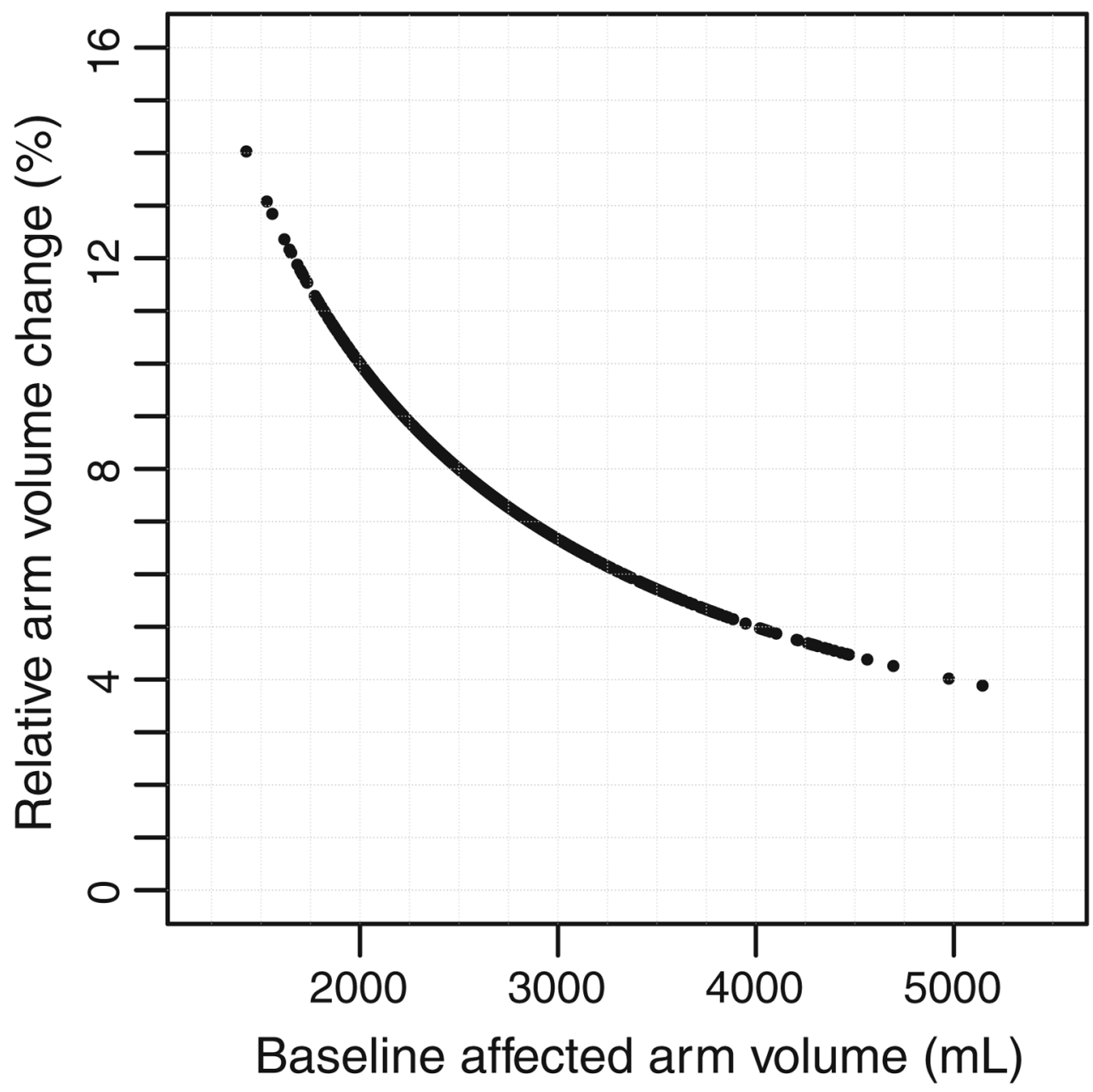

Fig. 2.

Relative arm volume change corresponding to arm volume increase by $200 \mathrm{~mL}$ in the unaffected arm of 677 patients 


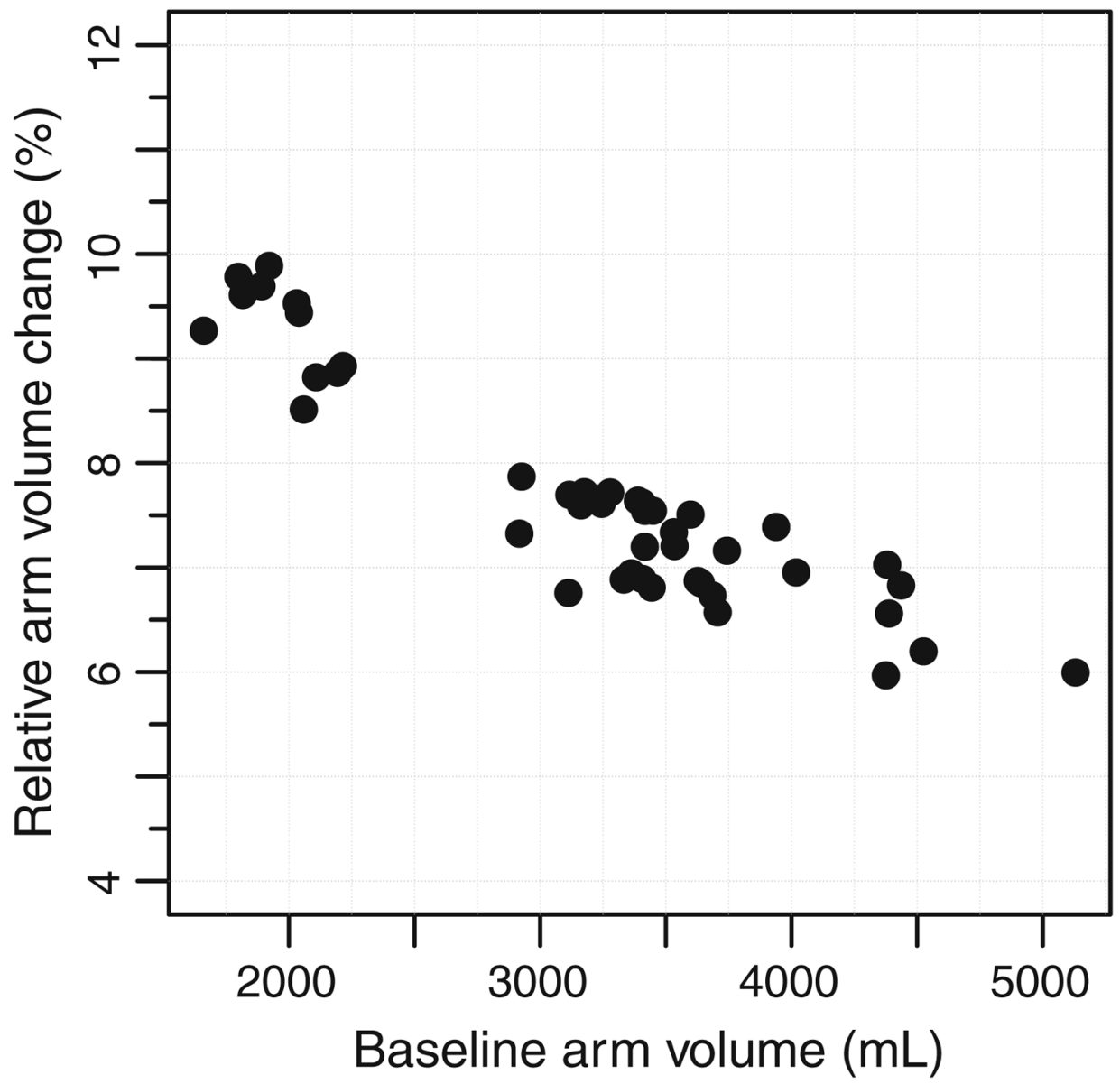

Fig. 3.

Predicted effect of a local $2 \mathrm{~cm}$ change in circumference of the unaffected arm compared to relative arm volume change in a sample of 45 patients 


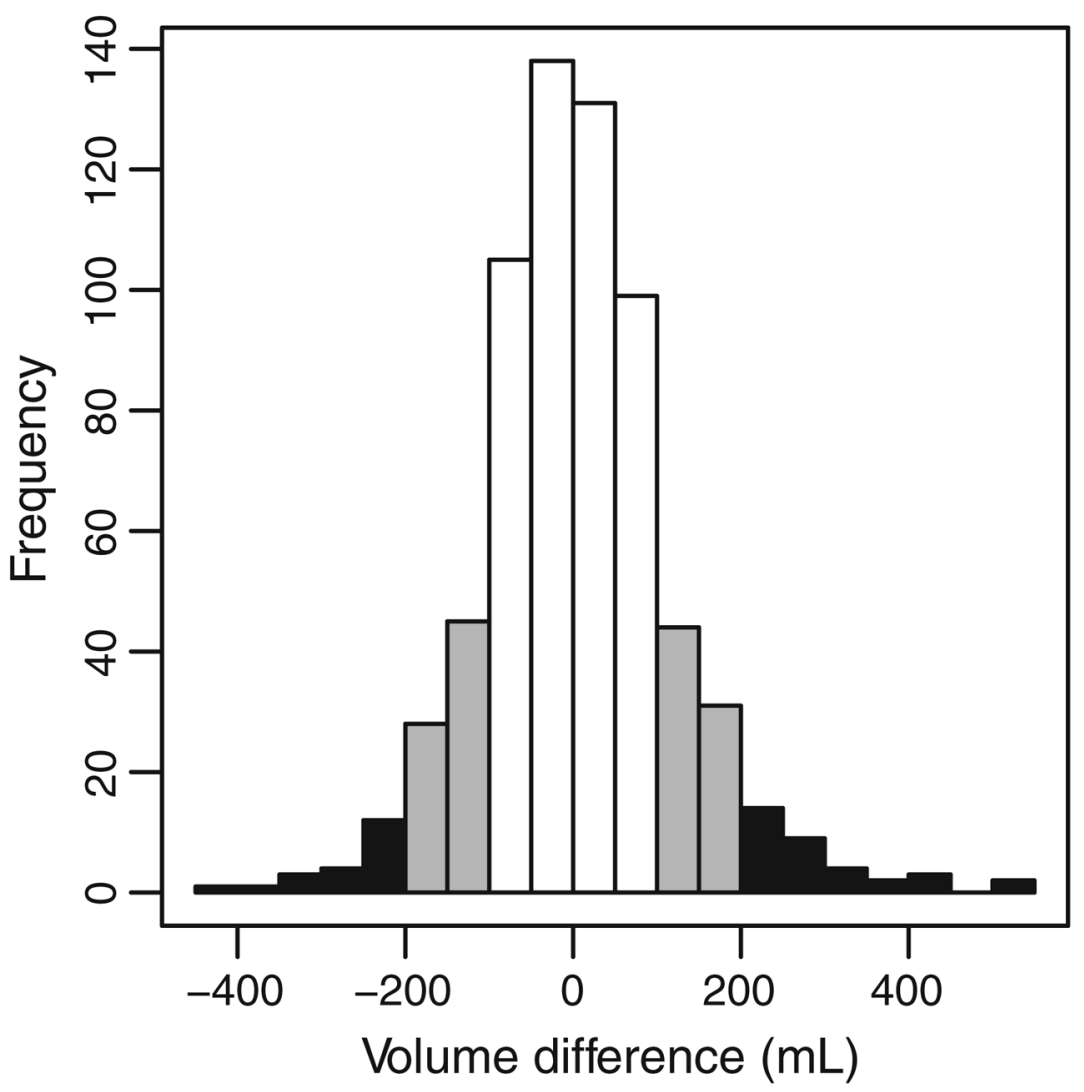

Fig. 4.

Histogram demonstrating absolute volume difference between arms for 677 patients at preoperative measurement. The black area corresponds to a difference of greater than $200 \mathrm{~mL}$, and the gray area corresponds to a difference of greater than $150 \mathrm{~mL}$ 


\section{Table 1}

Breast cancer (BC)-related lymphedema (LE) randomized trials

\begin{tabular}{|c|c|c|c|c|}
\hline Reference & Years & Sample size & Measurement technique & Definition of LE \\
\hline Devoogdt [38] & 2011 & $160 \mathrm{BC}$ patients & Water displacement, tape circumferences & $\begin{array}{l}\geq 200 \mathrm{~mL} \text { by water displacement } \\
\geq 2 \mathrm{~cm} \text { increase }\end{array}$ \\
\hline Kim [39] & 2010 & $40 \mathrm{BC}$ patients $\mathrm{w} / \mathrm{LE}$ & Tape circumferences, lymphoscintigraphy & $\begin{array}{l}>2 \mathrm{~cm} \text { difference between arms, } \mathrm{LE} \\
\text { diagnosis by lymphoscintigraphy }\end{array}$ \\
\hline Ashikaga [45] & 2010 & 3,983 BC patients & Water displacement & $\begin{array}{l}\geq 10 \% \text { relative arm volume difference } \\
\text { between arms }\end{array}$ \\
\hline Omar [40] & 2010 & $50 \mathrm{BC}$ patients w/LE & Tape circumferences & $\begin{array}{l}>2 \mathrm{~cm} \text { and }<8 \mathrm{~cm} \text { difference between } \\
\text { arms }\end{array}$ \\
\hline Smith [48] & 2009 & $643 \mathrm{BC}$ patients & Tape circumferences & Average $\%$ increase in arm volume \\
\hline Kozanoglu [41] & 2009 & $47 \mathrm{BC}$ patients w/LE & Tape circumferences & $\geq 2 \mathrm{~cm}$ at $3 / 7$ points of affected arm \\
\hline Schmitz [31] & 2010 & $154 \mathrm{BC}$ patients & Water displacement, CTC standards & $\geq 10 \%$ volume difference between arms \\
\hline Lucci [37] & 2007 & $\begin{array}{l}891 \text { BC patients, } 821 \mathrm{w} / \\
\text { LE }\end{array}$ & Tape circumferences & $\geq 2 \mathrm{~cm}$ difference between arms \\
\hline Ahmed [3] & 2006 & $45 \mathrm{BC}$ patients & Tape circumferences & $\begin{array}{l}\geq 2 \mathrm{~cm} \text { circumference difference } \\
\text { between arms }\end{array}$ \\
\hline
\end{tabular}

$S L N$ sentinel lymph node, $A L N D$ axillary lymph node dissection 